\title{
GAMMA-RAY OPTICAL COUNTERPART SEARCH EXPERIMENT \\ (GROCSE)
}

\author{
Carl Akerlof, Marco Fatuzzo, Brian Lee \\ University of Michigan, Ann Arbor, MI 48109 \\ Richard Bionta, Arno Ledebuhr, Hye-Sook Park \\ Lawrence Livermore National Laboratory, Livermore, CA 94550 \\ Scott Barthelmy, Thomas Cline, Neil Gehrels \\ NASA/Goddard Space Flight Center, Greenbelt, MD 20771
}

\begin{abstract}
The requirements of a gamma-ray burst optical counterpart detector are reviewed. By taking advantage of real-time notification of bursts, new instruments can make sensitive searches while the gamma-ray transient is still in progress. A wide field of view camera at Livermore National Laboratories has recently been adapted for detecting GRB optical counterparts to a limiting magnitude of 8 . A more sensitive camera, capable of reaching $m_{v}=14$, is under development.
\end{abstract}

\section{INTRODUCTION}

The persistent mystery of the origins of the gamma-ray burst phenomena has escalated the interest in searching for counterparts at other wavelengths. Since follow-up observations with conventional radio and optical telescopes require relatively small error boxes, the BATSE burst locations by themselves have not yet led to detections at these longer wavelengths. Data from various Interplanetary Network satellite configurations has provided much better localization but the time delays to acquire and process the results is at least 8 hours. ${ }^{1}$ With a response time of days following the event, no reliable optical counterparts have been found for GRBs down to a limiting magnitude of $m_{v}=24$ with the exception of a few soft gamma ray repeaters. ${ }^{2}$ We wish to radically reduce this response time to seconds by making use of the BACODINE GRB early warning system ${ }^{3}$ developed by Scott Barthelmy at Goddard. Charles Meegan in the introductory talk ${ }^{4}$ for this workshop showed a histogram of the durations of BATSE events (see figure 1). The median event durations are approximately 6 seconds for $50 \%$ of the flux $\left(\tau_{50}\right)$ and 20 seconds for $90 \%\left(\tau_{90}\right)$. The goal of the GROCSE experiment is to aim a sensitive wide field of view camera to estimated burst coordinates within these time scales.

No reliable theories have attempted signal detection limits to compute the optical luminosity of GRBs. Since there are no Arabic names for these phenomena, we can immediately infer a limiting visual magnitude of the order of 2 or more. Recent results from the ETC detector ${ }^{5}$ push the limit to at least 6 . Since 


\section{Gamma-Ray Optical Counterpart Search}

the intrinsic detection limit for such an optical system is set by the brightness of the night sky, we can roughly estimate the performance of a detector from the following approximate relations:

Night sky photon rate per pixel:

$$
N_{\text {sky }}=9 \times 10^{7} \frac{\pi}{4} \frac{\text { pixel area }}{f^{2}} \text { photons } / \text { pixel }- \text { sec }
$$

where pixel area is given in $\mathrm{cm}^{2}$ and $f$ is the focal length/aperture ratio.

Photon flux from a star with magnitude $m_{v}$ :

$$
N\left(m_{v}\right)=10^{6}(2.51189)^{-m_{v}} \text { photons } / \mathrm{cm}^{2}-\mathrm{sec}
$$

If all the starlight falls within a single CCD pixel, the $5 \sigma$ detection limit is given by:

$$
m_{v}=1.086 \log \left(18.7 \cdot f \cdot D^{2} \sqrt{\frac{\eta t}{\text { pixel area }}}\right)
$$

where $\eta$ is the detector efficiency, $t$ is the exposure time in seconds, and $D$ is the lens aperture in $\mathrm{cm}$.

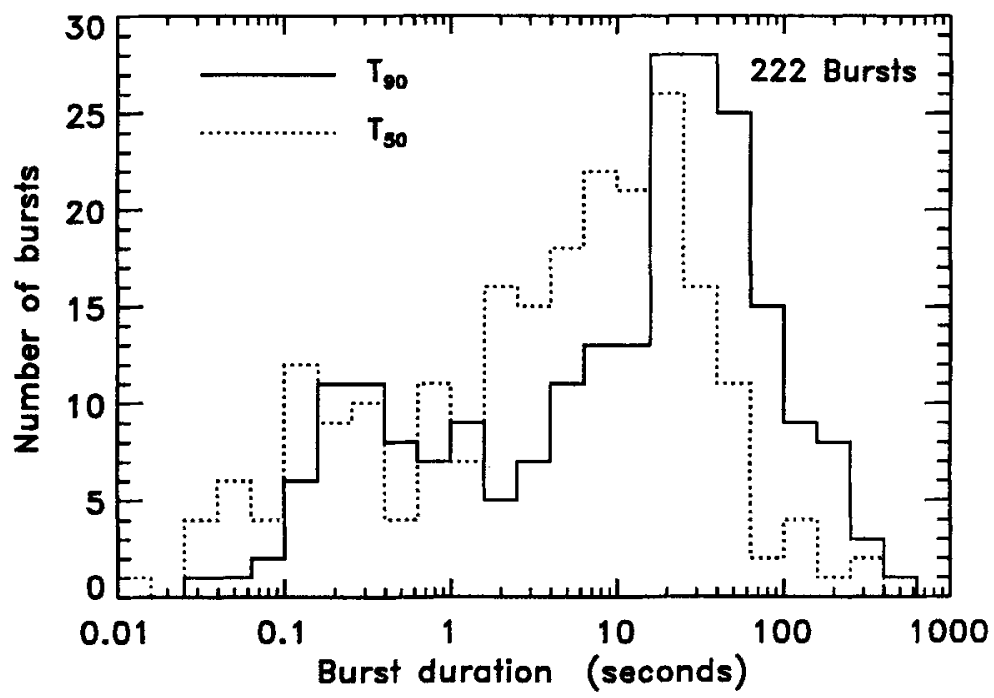

Figure 1. BATSE burst duration distribution (from C. Meegan, et al.)

Certainly this formula is not expected to predict the absolute performance level of a single system but for similar image sensor technology it should enable comparison of two different optical systems. From the known performance of the ETC detector, it appears possible to reach a magnitude $m_{v}=14$ sensitivity with a lens aperture of $89 \mathrm{~mm}$ and moderate $f$ number. 
A limiting visual magnitude of 14 is an interesting benchmark because it defines a power level of about $10^{-11} \mathrm{ergs} / \mathrm{cm}^{2}-\mathrm{sec}$ at the Earth. Since the brightest GRBs have been detected with energy fluxes of the order of $10^{-4}$ ergs $/ \mathrm{cm}^{2}, m_{v}=14$ corresponds to an optical X-ray $/ \gamma$-ray power ratio of $10^{-6}$, assuming a typical pulse duration of 10 seconds. This is also roughly the ratio of the quantum energies of the photons. In the absence of any guidance from a theory of gamma-ray bursts, this seems like a useful goal to aim towards.

The maximum event rate can be derived directly from the BATSE value of about 0.8 events per day. By using the GRB coordinates supplied by the BACODINE system, we should be able to cover $\pi$ steradians of sky with an average duty cycle of $10 \%$, averaged over a year. This corresponds to 0.02 events/day or 7 events/year.

\begin{tabular}{|c|c|}
\hline focal length & $250 \mathrm{~mm}$ \\
\hline aperture & $89 \mathrm{~mm}(f 2.8)$ \\
\hline imaged FOV & 0.621 steradians \\
\hline image reduction & $3.8: 1$ \\
\hline \multicolumn{2}{|r|}{23 image intensifier - CCD sensors } \\
\hline $\mathrm{CCDs}$ & $384 \times 576$ Thomson \\
\hline pixel size & $23 \mu \times 23 \mu$ \\
\hline pixel coverage & 1.2 arc-min \\
\hline exposure time & $0.1-1.0 \mathrm{sec}$ \\
\hline camera mount & Contraves inertial guidance test system \\
\hline slew rate & $100^{\circ} / \mathrm{sec} ; 200^{\circ} / \mathrm{sec}$ \\
\hline limiting magnitude & 8.0 \\
\hline Response time & $\begin{array}{lr}\text { BATSE } \rightarrow \text { GSFC } & 4.5 \text { secs } \\
\text { GSFC } \rightarrow \text { LLNL } & 2.7 \text { secs } \\
\text { Camera slewing } & \sim 5.0 \text { secs }\end{array}$ \\
\hline
\end{tabular}

To begin our search for optical counterparts, the GROCSE collaboration has adapted a wide field of view camera which was originally designed for the Strategic Defense Initiative program (SDI). The essential element of this camera is a wide field lens of a rather unusual design. All surfaces are spherically concentric, leading to a similarly shaped focal surface, as shown in figure 2. The SDI application required rapid frame rates so that the focal surface is divided into 23 rectangular segments, each of which is coupled via a coherent fiber optic bundle to an image intensifier followed by a second fiber optical bundle interfaced 


\section{Gamma-Ray Optical Counterpart Search}

to a CCD. The disadvantage of such a system is a fairly poor overall quantum efficiency. The salient characteristics of this camera are listed in Table I. A schematic drawing of the camera is depicted in figure 3.

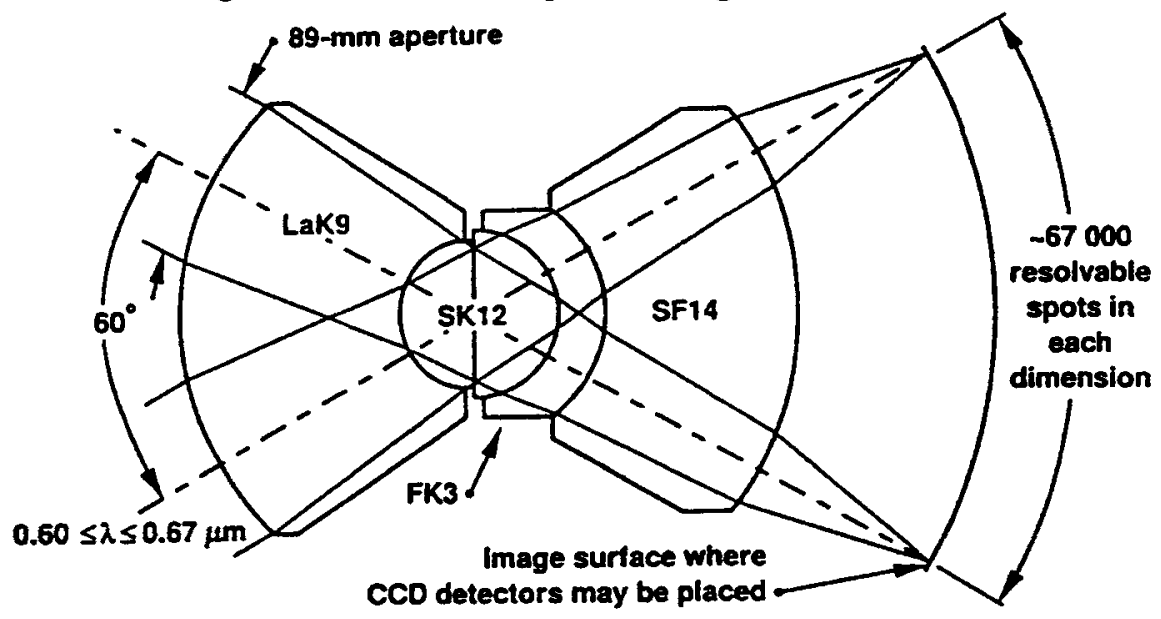

\section{$100 \mathrm{~mm}$}

Figure 2. Lens design for the Livermore $89 \mathrm{~mm}$ diameter wide-field-of-view camera.

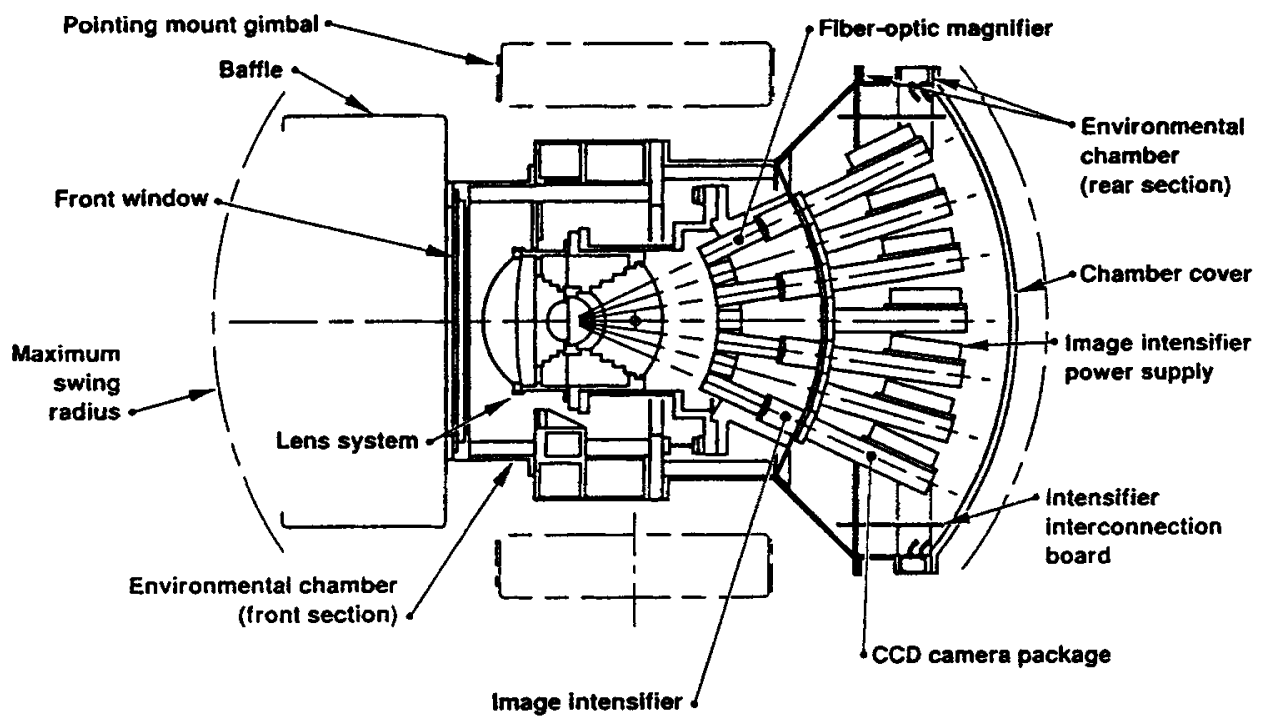

Figure 3. Schematic diagram of the Livermore widefield-of-view camera imaging system. 
The camera is mounted in a Contraves alti-azimuth inertial guidance test system capable of accurate and rapid slewing to a designated celestial coordinate. The total response time to a BATSE burst is given by the sum of three numbers: 4.5 seconds from burst detection onboard GRO to coordinate computation at Goddard, 2.7 seconds to send a burst coordinate packet via Internet to Livermore, and about 5 seconds to slew the camera. This sum of about 12 seconds is approximately half of the $\tau_{90}$ burst duration.

Data taking, using the BATSE-generated trigger system, has just begun. The main task we now face is developing the image processing software that can identify transient stellar-like objects against a background of satellite tracks. Comparison of star images with the SAO catalog shows that we are operating with a limiting magnitude of about 8 . During the next 12 months, we expect to bring the current wide field camera into routine operation, taking observations whenever weather permits. At the same time, we are also developing a prototype system using similar optics but with considerably improved imaging quantum efficiency. With such a second generation optical detector, we hope to attain the threshold sensitivity required to finally identify GRBs at optical wavelengths.

\section{REFERENCES}

1. Kevin Hurley, this proceedings

2. Bradley Schaefer, this proceedings

3. Scott Barthelmy, this proceedings

4. Charles Meegan, this proceedings

5. Hans Krimm, this proceedings 